\title{
Interdependence in fuzzy multiple objective programming *
}

\author{
Christer Carlsson \\ ccarlsso@abo.fi
}

\author{
Robert Fullér \\ rfuller@abo.fi
}

\begin{abstract}
In multiple objective programs [MOP], application functions are established to measure the degree of fulfillment of the decision maker's requirements (achievement of goals, nearness to an ideal point, satisfaction, etc.) about the objective functions (see e.g. [7, 24]) and are extensively used in the process of finding "good compromise" solutions. In [6] we demonstrated that the use of interdependences among objectives of a MOP in the definition of the application functions provides for more correct solutions and faster convergence. In this paper, generalizing the principle of application functions to fuzzy multiple objective programs [FMOP] with interdependent objectives, we define a large family of application functions for FMOP and illustrate our ideas by a simple three-objective program.
\end{abstract}

\section{Introduction}

Decision making with interdependent multiple criteria is a surprisingly difficult task. If we have clearly conflicting objectives there normally is no optimal solution which would simultaneously satisfy all the criteria. On the other hand, if we have pair-wisely supportive objectives, such that the attainment of one objective helps us to attain another objective, then we should exploit this property in order to find effective optimal solutions. In some earlier work $[4,5]$ we used the fuzzy Pareto optimal set of non-dominated alternatives as a basis for an OWA-type operator [21, 22], which was used to rank-order the alternatives according to an effective attainment of the interdependent criteria. This turned out to be a promising approach, and it will be explored and further developed in this paper. Decision making is possible and required if there is a set $X$ of available decision alternatives $x_{i}(i \geq 2)$; if we then introduce DM, a well-defined (in a utility theory sense) decision maker, and a real-valued function $c(x)$ it is possible to use preferences as a basis for the selection of a decision alternative ([19]). DM is said to prefer $x_{i}$ over $x_{k}$ (both in $X$ ) iff $c\left(x_{i}\right)>c\left(x_{k}\right)$, and DM is said to be indifferent between $x_{i}$ and $x_{k}$ iff $c\left(x_{i}\right)=c\left(x_{k}\right)$. If we consider the set $X$, and assume that the function $c(x)$ has some rather normal properties (cf. [17]), we have the following well-defined problem: to find an $x^{*} \in X$ such that $c\left(x^{*}\right)>c\left(x_{i}\right)$ for all $x_{i} \in X$. As this is a question of simple enumeration as soon as the problem has been formulated, no actual decision making is involved at this stage (the selection/definition of $x$ and $c(x)$ actually requires decision making, but of quite another kind). Consider now, ceteris paribus, the case when the preferences of DM rest on

$$
c(x)=\left(c_{1}(x), \ldots, c_{m}(x)\right)
$$

and the ranking of the decision alternatives $x_{i}$ and $x_{k}(\in X)$ is not well-defined anymore (cf. [17]). The most we can do is to organise alternatives according to efficiency or non-dominance. Consider the

\footnotetext{
${ }^{*}$ The final version of this paper appeared in: C. Carlsson and R. Fullér, Interdependence in fuzzy multiple objective programming Fuzzy Sets and Systems 65(1994) 19-29. doi: 10.1016/0165-0114(94)90244-5
} 
alternative $x_{i}$; it is said to be strongly efficient or Pareto optimal (cf. [5]) iff it is impossible to find an $x_{k}(k \neq i)$ such that $c_{j}\left(x_{k}\right) \geq c_{j}\left(x_{i}\right)$ for all $j=(1, \ldots, m)$ and $c_{j}\left(x_{k}\right)>c_{j}\left(x_{i}\right)$ for at least one $j$.

There are numerous methods available for finding $x_{i}$ ( $\mathrm{cf}$. $[11,23,25]$ ), if (i) the set of feasible alternatives is well-defined, (ii) there is a rationally structured model of preferences, where the preferences satisfy some necessary mathematical properties, and (iii) the problem of finding an efficient alternative is a well-formulated mathematical problem [14].

If the criteria are independent, there are various aggregation methods (additive forms, weighted sums, expected utility, and utility additive and multiplicative forms) available for comparing and ranking nondominated alternatives, which (in a sense) reduces the multiple criteria problem to a situation comparable with the single criterion decision - and quite a few insights in the decision problem are simplified away.

In a recent paper Sakawa and Yano [17] very nicely demonstrate the state of the art when we want to deal with multiple criteria problems which are - and cannot be - well-defined. Their model is a multiple objective linear fractional programming model, with fuzzy parameters and an uncertain goal for the objective function. The uncertainty is of two types: (i) an uncertainty of the satisfaction with the value of an objective function; and (ii) an uncertainty of the possibility to generate the wanted value of the objective function. The Sakawa-Yano method handles both types of uncertainties and reduces the problem to an ordinary multiobjective programming problem.

The $I$ - $\gamma$-Pareto optimality is introduced as a new solution concept; the optimality denotes the agreement between the multiobjective fuzzy linear fractional function and the fuzzy goal.

Another approach has been developed by Kacprzyk and Yager (cf. [11]), in which they use fuzzy logic with linguistic qualifiers to bring human consistency to multiobjective decision making. They use rather a nonconventional solution concept, which is based on searching for some optimal option which "best satisfies most of the important objectives"; this differs from the traditional notion to try to find an optimal option which best satisfies "all the objectives".

Both the Sakawa-Yano and the Kacprzyk-Yager papers seem to support the idea that traditional MCDMmodels, and their underlying notion of an optimal solution, are much too limited for actual, real-world problem-solving with MCDM methods. The reason for this is simple: when the solution derived from a well-formulated mathematical MCDM-model is applied to an actual problem there are some major problems to consider (cf. [14]): (i) the set of feasible decision alternatives is fuzzy, and this set changes during the problem solving process; (ii) the DM does not exist as an active entity, and the preferences consist of badly formulated beliefs, which are riddled with conflicts and contradictions; (iii) data on preferences are imprecise, and (iv) a decision should be good or bad not only in relation to some model, but in relation to the actual context. These problems have initiated active and fast-growing research on the use of fuzzy set theory in solving multiple criteria decision problems [1, 2, 3, 15, 16, 20, 25].

Much more interesting, and much closer to multiple criteria problems in the real world, than the traditional MCDM-problems are the cases with interdependent (conflicting, supportive) criteria. Consider a decision problem in which we have to find an $x^{*} \in X$ such that three different criteria $c_{1}, c_{2}$ and $c_{3}$ are all satisfied when $c_{1}$ and $c_{2}$ support each others, $c_{2}$ and $c_{3}$ are conflicting, and $c_{3}$ and $c_{1}$ are supportive (with respect to some directions).

It is clear that the choice is rather a complex process unless we find some systematic ways to evaluate and explore the interdependence; nevertheless, it is quite a common case in fairly standard business situations (cf. e.g. [5]). The evaluation is probably more straightforward for a single decisin maker than for a group of decision makers, each of which is defending one or two of the criteria. In problems with interdependent multiple criteria we do not have to handle just uncertainty and imprecision, there is an added element of inability to grasp the consequences of the interdependencies; this is also a field of research in which the use of models based on fuzzy set theory should give us decisive advantages. 
In Section 2 we will introduce application functions and show how they can be applied in multiple objective programming models; the application functions are further developed to fuzzy multiple objective programming in Section 3, in which we show how to deal with the problem of interdependence; the method is illustrated with a simple example in Section 4 and the results are summarized in Section 5.

\section{Application functions for MOP}

Consider a multiple objective program

$$
\max _{x \in X}\left\{f_{1}(x), \ldots, f_{k}(x)\right\}
$$

where $f_{i}: \mathbb{R}^{n} \rightarrow \mathbb{R}$ are objective functions, $x \in \mathbb{R}^{n}$ is the decision variable, and $X$ is a subset of $\mathbb{R}^{n}$ without any additional conditions for the moment.

An application function $h_{i}$ for MOP (1) is defined as [7, 24]

$$
h_{i}: \mathbb{R} \rightarrow[0,1]
$$

such that $h_{i}(t)$ measures the degree of fulfillment of the decision maker's requirements about the $i$-th objective by the value $t$. In other words, with the notation of

$$
H_{i}(x)=h_{i}\left(f_{i}(x)\right)
$$

$H_{i}(x)$ may be considered as the degree of membership of $x$ in the fuzzy set "good solutions" for the $i$-th objective. Then a "good compromise solution" to (1) may be defined as an $x \in X$ being "as good as possible" for the whole set of objectives. Taking into consideration the nature of $H_{i}(),. i=1, \ldots k$, it is quite reasonable to look for such a kind of solution by means of the following auxiliary problem

$$
\max _{x \in X}\left\{H_{1}(x), \ldots, H_{k}(x)\right\}
$$

For $\max \left\{H_{1}(x), \ldots, H_{k}(x)\right\}$, which may be interpreted as a synthetical notation of a conjuction statement ("maximize jointly all objectives") and $H_{i}(x) \in[0,1]$, it is reasonable to use a t-norm $T$ [18] to represent the connective AND. In this way (2) turns into the single-objective problem

$$
\max _{x \in X} T\left(H_{1}(x), \ldots, H_{k}(x)\right) .
$$

There exist several ways to introduce application functions [11]. Usually, the authors consider increasing membership functions (the bigger is better) of the form

$$
h_{i}(t)= \begin{cases}1 & \text { if } t \geq M_{i} \\ v_{i}(t) & \text { if } m_{i} \leq t \leq M_{i} \\ 0 & \text { if } t \leq m_{i}\end{cases}
$$

where $m_{i}\left[\geq \min _{x \in X} f_{i}(x)\right]$ denotes the reservation (or security) level representing minimal requirement and $M_{i}\left[\leq \max _{x \in X} f_{i}(x)\right]$ is the desirable level on the $i$-th objective.

In [6] we have introduced measures of interdependences between the objectives, in order to provide for a better understanding of the decision problem, and to find effective and more correct solutions to MOP (1).

Definition 2.1 [6] Let $f_{i}$ and $f_{j}$ be two objective functions of (1). We say that 
(i) $f_{i}$ supports $f_{j}$ on $X$ (denoted by $f_{i} \uparrow f_{j}$ ) if $f_{i}\left(x^{\prime}\right) \geq f_{i}(x)$ entails $f_{j}\left(x^{\prime}\right) \geq f_{j}(x)$, for all $x^{\prime}, x \in X$;

(ii) $f_{i}$ is in conflict with $f_{j}$ on $X$ (denoted by $f_{i} \downarrow f_{j}$ ) if $f_{i}\left(x^{\prime}\right) \geq f_{i}(x)$ entails $f_{j}\left(x^{\prime}\right) \leq f_{j}(x)$, for all $x^{\prime}, x \in X$;

(iii) $f_{i}$ and $f_{j}$ are independent on $X$, otherwise.

Figure 1: A typical example of conflict on $\mathbb{R}$.

Figure 2: Supportive functions on $\mathbb{R}$

Let $f_{i}$ be an objective function of (1). In [6] we defined the grade of interdependency, denoted by $\Delta\left(f_{i}\right)$, of $f_{i}$ as

$$
\Delta\left(f_{i}\right)=\sum_{f_{i} \uparrow f_{j}, i \neq j} 1-\sum_{f_{i} \downarrow f_{j}} 1, \quad i=1, \ldots, k .
$$

If $\Delta\left(f_{i}\right)$ is positive and large then $f_{i}$ supports a majority of the objectives, if $\Delta\left(f_{i}\right)$ is negative and large then $f_{i}$ is in conflict with a majority of the objectives, if $\Delta\left(f_{i}\right)$ is positive and small then $f_{i}$ supports more objectives than it hinders, and if $\Delta\left(f_{i}\right)$ is negative and small then $f_{i}$ hinders more objectives than it supports. Finally, if $\Delta\left(f_{i}\right)=0$ then $f_{i}$ is independent from the others or supports the same number of objectives as it hinders.

In [6] we used explicitely the interdependences in the solution method. Namely, first we defined $H_{i}$ as

$$
H_{i}(x)= \begin{cases}1 & \text { if } f_{i}(x) \geq M_{i} \\ 1-\frac{M_{i}-f_{i}(x)}{M_{i}-m_{i}} & \text { if } m_{i} \leq f_{i}(x) \leq M_{i} \\ 0 & \text { if } f_{i}(x) \leq m_{i}\end{cases}
$$

i.e. all membership functions are defined to be linear.

Then we computed $\Delta\left(f_{i}\right)$ for $i=1, \ldots, k$, and changed the shapes of $H_{i}$ according to the value of $\Delta\left(f_{i}\right)$ as follows

(1) If $\Delta\left(f_{i}\right)=0$ then we did not change the shape.

(2) If $\Delta\left(f_{i}\right)>0$ then instead of the linear membership function we used

$$
H_{i}\left(x, \Delta\left(f_{i}\right)\right)= \begin{cases}1 & \text { if } f_{i}(x) \geq M_{i} \\ \left(1-\frac{M_{i}-f_{i}(x)}{M_{i}-m_{i}}\right)^{1 /\left(\Delta\left(f_{i}\right)+1\right)} & \text { if } m_{i} \leq f_{i}(x) \leq M_{i} \\ 0 & \text { if } f_{i}(x) \leq m_{i}\end{cases}
$$

(3) If $\Delta\left(f_{i}\right)<0$ then instead of the linear membership function we used

$$
H_{i}\left(x, \Delta\left(f_{i}\right)\right)= \begin{cases}1 & \text { if } f_{i}(x) \geq M_{i} \\ \left(1-\frac{M_{i}-f_{i}(x)}{M_{i}-m_{i}}\right)^{\left|\Delta\left(f_{i}\right)\right|+1} & \text { if } m_{i} \leq f_{i}(x) \leq M_{i} \\ 0 & \text { if } f_{i}(x) \leq m_{i}\end{cases}
$$

Figure 3: The case of linear membership function. 
Figure 4: $H_{i}\left(x, \Delta\left(f_{i}\right)\right)$ if $\Delta\left(f_{i}\right)>0$.

Figure 5: $H_{i}\left(x, \Delta\left(f_{i}\right)\right)$ if $\Delta\left(f_{i}\right)<0$.

Then we solved the following auxiliary problem

$$
\max _{x \in X} T\left(H_{1}\left(x, \Delta\left(f_{1}\right)\right), \ldots, H_{k}\left(x, \Delta\left(f_{k}\right)\right)\right)
$$

Let us suppose that we have a decision problem with many $(k \geq 7)$ objective functions. It is clear (due to the interdependences between the objectives), that we find optimal compromise solutions rather closer to the values of independent minima than maxima.

The basic idea of introducing the shape functions can be explained then as follows: if we manage to increase the value of the $i$-th objective having a large positive $\Delta\left(f_{i}\right)$ then it entails the growth of the majority of criteria (because it supports the majority of the objectives), so we are getting essentially closer to the optimal value of the scalarizing function (because the losses on the other objectives are not so big, due to their definition).

\section{Application functions for FMOP}

Fuzzy sets of the real line are called fuzzy quantities. A fuzzy number $\tilde{a}$ is a fuzzy quantity with a continuous, finite-supported, fuzzy-convex and normalized membership function $\tilde{a}: \mathbb{R} \rightarrow[0,1]$.

The family of all fuzzy numbers will be denoted by $\mathcal{F}(\mathbb{R})$. An $\alpha$-level set of a fuzzy quantity $\tilde{a}$ is a non-fuzzy set denoted by $[\tilde{a}]^{\alpha}$ and is defined by

$$
[\tilde{a}]^{\alpha}=\{t \in \mathbb{R} \mid \tilde{a}(t) \geq \alpha\}
$$

for $\alpha \in(0,1]$ and $[\tilde{a}]^{\alpha}=\operatorname{cl}(\operatorname{supp} \tilde{a})$ for $\alpha=0$. A triangular fuzzy number $\tilde{a}$ denoted by $(a, \alpha, \beta)$ is defined as $\tilde{a}(t)=1-|a-t| / \alpha$ if $|a-t| \leq \alpha, \tilde{a}(t)=1-|a-t| / \beta$ if $|a-t| \leq \beta$ and $\tilde{a}(t)=0$ otherwise, where $a \in \mathbb{R}$ is the modal value and $\alpha, \beta$ are the left and right spreads of $\tilde{a}$, respectively.

Let $\tilde{a}, \tilde{b} \in \mathcal{F}(\mathbb{R})$ with $[\tilde{a}]^{\alpha}=\left[a_{1}(\alpha), a_{2}(\alpha)\right],[\tilde{b}]^{\alpha}=\left[b_{1}(\alpha), b_{2}(\alpha)\right]$ and let $\omega: \mathcal{F}(\mathbb{R}) \rightarrow \mathbb{R}$ be a defuzzyfier in $\mathcal{F}(\mathbb{R})$.

We suppose that crisp inequality relations between fuzzy numbers are defined by defuzzifiers, i.e.

$$
\tilde{a} \leq \tilde{b} \text { iff } \Omega(\tilde{a}) \leq \Omega(\tilde{b}) .
$$

We metricize $\mathcal{F}(\mathbb{R})$ by the metrics [10],

$$
D_{p}(\tilde{a}, \tilde{b})=\left(\int_{0}^{1} d\left([\tilde{a}]^{\alpha},[\tilde{b}]^{\alpha}\right)^{p}\right)^{1 / p}
$$

for $1 \leq p \leq \infty$, especially, for $p=\infty$ we get

$$
D_{\infty}(\tilde{a}, \tilde{b})=\sup _{\alpha \in[0,1]} d\left([\tilde{a}]^{\alpha},[\tilde{b}]^{\alpha}\right),
$$

where $d$ denotes the classical Hausdorff metric in the family of compact subsets of $\mathbb{R}^{2}$. We shall use the following inequality relation between fuzzy numbers [9]

$$
\tilde{a} \leq \tilde{b} \text { iff }
$$




$$
\Omega(\tilde{a})=\int_{0}^{1} r\left(a_{1}(r)+a_{2}(r)\right) d r \leq \Omega(\tilde{b})=\int_{0}^{1} r\left(b_{1}(r)+b_{2}(r)\right) d r
$$

Consider a fuzzy multiple objective program [FMOP]

$$
\max _{x \in X}\left\{\tilde{f}_{1}(x), \ldots, \tilde{f}_{k}(x)\right\}
$$

where $\tilde{f}_{i}: \mathbb{R}^{n} \rightarrow \mathcal{F}(\mathbb{R})$ (i.e. a fuzzy-number-valued function) and $X \subset \mathbb{R}^{n}$.

An application function for FMOP (6) is defined as

$$
h_{i}: \mathcal{F}(\mathbb{R}) \rightarrow[0,1]
$$

such that $h_{i}(\tilde{t})$ measures the degree of fulfillment of the decision maker's requirements about the $i$-th objective by the (fuzzy number) value $\tilde{t}$. In other words, with the notation of

$$
H_{i}(x)=h_{i}\left(\tilde{f}_{i}(x)\right)
$$

$H_{i}(x)$ may be considered as the degree of membership of $x$ in the fuzzy set "good solutions" for the $i$-th fuzzy objective.

To construct such application functions for FMOP problems is usually not an easy task.

Suppose that we have two reference points from $\mathcal{F}(\mathbb{R})$, denoted by $\tilde{m}_{i}$ and $\tilde{M}_{i}$, which represent undesired and desired levels for each objective function $\tilde{f}_{i}$. We can now state (6) as follows: find an $x^{*} \in X$ such that $\tilde{f}_{i}\left(x^{*}\right)$ is as close as possible to the desired point $\tilde{M}_{i}$ and as far as possible from the undisered point $\tilde{m}_{i}$, for each $i=1, \ldots, k$.

We suggest the use of the following family of application functions

$$
H_{i}(x)=\min \left\{1-\frac{1}{1+D\left(\tilde{m}_{i}, \tilde{f}_{i}(x)\right)}, \frac{1}{1+D\left(\tilde{M}_{i}, \tilde{f}_{i}(x)\right)}\right\}
$$

or, more generally,

$$
H_{i}(x)=T\left(1-\frac{1}{1+D\left(\tilde{m}_{i}, \tilde{f}_{i}(x)\right)}, \frac{1}{1+D\left(\tilde{M}_{i}, \tilde{f}_{i}(x)\right)}\right)
$$

where $T$ is a t-norm, $D$ is a metric (e.g $\left.D_{p}\right)$ in $\mathcal{F}(\mathbb{R})$.

It is clear that the bigger the value of $H_{i}(x)$ the closer the value of the $i$-th objective function to the desired level or/and further from the undesired level, and vica versa the smaller the value of $H_{i}(x)$ the closer its value to the undesired level or/and further from the desired level.

In (7) the t-norm $T$ measures the degree of satisfaction of two (conflicting) goals "to be far from the undesired point and to be close to the desired point". The particular t-norm $T$ should be chosen very carefully, because it can occur that $H_{i}(x)$ attends its maximal value at a point which is very far from the undesired point, but not close enough to the desired point.

For example, if $T$ is the weak t-norm $(T(x, y)=\min \{x, y\}$ if $\max \{x, y\}=1$ and $T(x, y)=0$ otherwise) then $H_{i}(x)$ positive if and only if $\tilde{f}_{i}(x)=\tilde{M}_{i}$, i.e. we have managed to reach completely the desired point, which is rarely the case, because $\tilde{M}_{i}$ is not necessarily in the range of $\tilde{f}_{i}$. Another crucial point is the relative setting of the desired and undesired points. If $D\left(\tilde{m}_{i}, \tilde{M}_{i}\right)$ is small then it is impossible to find an $x^{*} \in X$ satisfying the condition " $\tilde{f}_{i}(x)$ is close to $\tilde{M}_{i}$ and is far from $\tilde{m}_{i}$ ".

Another possibility to determine application functions for (6) is the use of defuzzifiers. Suppose that a defuzzifier $\Omega$ can be established, such that $\tilde{f}_{i}(x)$ can be considered as a fuzzy number representing the 
statement " $t$ is approximately equal to $\Omega\left(\tilde{f}_{i}(x)\right)$ " for each $x \in X$. Then instead of FMOP (6) we can consider directly its defuzzified version

$$
\max _{x \in X}\left\{\Omega\left(\tilde{f}_{1}(x)\right), \ldots, \Omega\left(\tilde{f}_{k}(x)\right)\right\} .
$$

Suppose that the decision maker wishes to gain on the $i$-th objective minimum approximately $m_{i}$, but he is satisfied when its value is approximately $M_{i}$ or bigger.

In this case we suggest the use of the family of application functions

$$
H_{i}(x)= \begin{cases}1 & \text { if } M_{i} \leq \Omega\left(\tilde{f}_{i}(x)\right) \\ \frac{\Omega\left(\tilde{f}_{i}(x)\right)-m_{i}}{M_{i}-m_{i}} & \text { if } m_{i} \leq \Omega\left(\tilde{f}_{i}(x)\right) \leq M_{i} \\ 0 & \text { if } \Omega\left(\tilde{f}_{i}(x)\right) \leq m_{i}\end{cases}
$$

Remark 3.1 It is easy to see that $D\left(\tilde{f}_{i}(x), \tilde{M}_{i}\right)$ is a better measure for the distance between the actual value and the desired value of the $i$-th objective function than the simple difference $\left|\Omega\left(\tilde{f}_{i}(x)\right)-\Omega\left(\tilde{M}_{i}\right)\right|$, because, depending on the definition of the defuzzifier $\Omega$, the later distance can be very small even if all $\alpha$-level sets of $\tilde{f}_{i}(x)$ and $\tilde{M}_{i}$ are very far from each other. For example, if we take $D_{\infty}$ for a metric and the defuzzifier $\Omega$ is defined by (5) then we have

$$
|\Omega(\tilde{a})-\Omega(\tilde{b})| \leq D_{\infty}(\tilde{a}, \tilde{b})
$$

for all $\tilde{a}, \tilde{b} \in \mathcal{F}(\mathbb{R})$.

Remark 3.2 It should be noted that the direct translation of (6) to (8) is correct only if the (fuzzy number) values of the objective functions can be interpreted as approximations of crisp numbers derived from a given defuzzifier $\Omega$.

Thus, similarly to the crisp case, FMOP (6) turns into the single-objective problem

$$
\max _{x \in X} T\left(H_{1}(x), \ldots, H_{k}(x)\right) .
$$

It is clear that the bigger the value of the objective function of problem (9) the closer the fuzzy functions are to their desired levels. Similarly to the crisp case, we shall modify the application functions, $H_{i}, i=$ $1, \ldots, k$ with respect to the interdependences among the objectives of FMOP (6).

We shall define the interdependences by the help of their application functions.

Definition 1 Let $\tilde{f}_{i}$ and $\tilde{f}_{j}$ be two objective functions of (6), and let $H_{i}$ and $H_{j}$ be the associated application functions. We say that

(i) $\tilde{f}_{i}$ supports $\tilde{f}_{j}$ on $X$ (denoted by $\left.\tilde{f}_{i} \uparrow \tilde{f}_{j}\right)$ if $H_{i}\left(x^{\prime}\right) \geq H_{i}(x)$ entails $H_{j}\left(x^{\prime}\right) \geq H_{j}(x)$ for all $x^{\prime}, x \in X$;

(ii) $\tilde{f}_{i}$ is in conflict with $\tilde{f}_{j}$ on $X$ (denoted by $\left.\tilde{f}_{i} \downarrow \tilde{f}_{j}\right)$ if $H_{i}\left(x^{\prime}\right) \geq H_{i}(x)$ entails $H_{j}\left(x^{\prime}\right) \leq H_{j}(x)$, for all $x^{\prime}, x \in X$;

(iii) $\tilde{f}_{i}$ and $\tilde{f}_{j}$ are independent on $X$, otherwise. 
Let $\tilde{f}_{i}$ be an objective function of (6) and let $H_{i}$ be its application function. We define the grade of interdependency, denoted by $\Delta\left(\tilde{f}_{i}\right)$, of $\tilde{f}_{i}$ as

$$
\Delta\left(\tilde{f}_{i}\right)=\sum_{H_{i} \uparrow H_{j}, i \neq j} 1-\sum_{H_{i} \downarrow H_{j}} 1, \quad i=1, \ldots, k .
$$

Then similarly to the crisp case, if $\Delta\left(\tilde{f}_{i}\right)$ is positive and large then $\tilde{f}_{i}$ supports a majority of the objectives, if $\Delta\left(\tilde{f}_{i}\right)$ is negative and large then $\tilde{f}_{i}$ is in conflict with a majority of the objectives, if $\Delta\left(\tilde{f}_{i}\right)$ is positive and small then $\tilde{f}_{i}$ supports more objectives than it hinders, and if $\Delta\left(\tilde{f}_{i}\right)$ is negative and small then $\tilde{f}_{i}$ hinders more objectives than it supports. Finally, if $\Delta\left(\tilde{f}_{i}\right)=0$ then $\tilde{f}_{i}$ is independent from the others or supports the same number of objectives as it hinders.

Remark 3.3 It is clear that interdependences among fuzzy objectives of (6) strongly depend on the definition of their application application functions. For example, if the application functions are defined in the sense of (7) then by altering the desired or/and undesired levels for the $i$-th objective function, it can modify $\Delta\left(\tilde{f}_{i}\right)$.

We use explicitely the interdependences in the solution method. Namely, first we change the shape of $H_{i}$ according to the value of $\Delta\left(\tilde{f}_{i}\right)$ as follows

(1) If $\Delta\left(\tilde{f}_{i}\right)=0$ then we do not change the shape, i.e $H_{i}\left(x, \Delta\left(\tilde{f}_{i}\right)\right):=H_{i}(x)$

(2) If $\Delta\left(\tilde{f}_{i}\right)>0$ then instead of $H_{i}(x)$ we take

$$
H_{i}\left(x, \Delta\left(\tilde{f}_{i}\right)\right):=H_{i}(x)^{1 /\left(\Delta\left(\tilde{f}_{i}\right)+1\right)}
$$

(3) If $\Delta\left(\tilde{f}_{i}\right)<0$ then instead of $H_{i}(x)$ we use

$$
H_{i}\left(x, \Delta\left(\tilde{f}_{i}\right)\right):=H_{i}(x)^{\left|\Delta\left(\tilde{f}_{i}\right)\right|+1}
$$

Then we solve the single objective problem

$$
\max _{x \in X} T\left(H_{1}\left(x, \Delta\left(\tilde{f}_{1}\right)\right), \ldots, H_{k}\left(x, \Delta\left(\tilde{f}_{k}\right)\right)\right)
$$

The basic idea of introducing this type of shape functions is the following: if we manage to increase the value of the $i$-th objective having a large positive $\Delta\left(\tilde{f}_{i}\right)$ then it entails the growth of the majority of criteria (because it supports the majority of the objectives), so we are getting essentially closer to the optimal value of the scalarizing function (because the losses on the conflicting objectives are not so big, due to their definition).

\section{Example}

We illustrate the use of applications functions by a simple three-objective program. Let us consider the following FMOP

$$
\max _{0 \leq x \leq 1}\left\{\tilde{f}_{1}(x), \tilde{f}_{2}(x), \tilde{f}_{3}(x)\right\}
$$

where

$$
\begin{aligned}
& \tilde{f}_{1}(x)=(x, 1 / 2,1 / 2), \\
& \tilde{f}_{2}(x)=\left(x^{2}, 1 / 2,1 / 2\right), \\
& \tilde{f}_{3}(x)=(1-x, 1 / 2,1 / 2) .
\end{aligned}
$$


(i.e. fuzzy numbers of triangular type with the same widths). Suppose that the decision maker has the following reference (desired and undesired) points

$$
\begin{aligned}
& \tilde{M}_{1}=(1,1 / 2,1 / 2), \tilde{m}_{1}=(0,1 / 2,1 / 2) \\
& \tilde{M}_{2}=(1,1 / 2,1 / 2), \tilde{m}_{2}=(0,1 / 2,1 / 2) \\
& \tilde{M}_{3}=(0,1 / 2,1 / 2), \tilde{m}_{3}=(1,1 / 2,1 / 2)
\end{aligned}
$$

Suppose further that we use $D_{\infty}$ to measure the distance between the actual level and the reference points, and choose the product t-norm in (7) and the minimum-norm in (11). Then we have

$$
\begin{gathered}
D\left(\tilde{m}_{1}, \tilde{f}_{1}(x)\right)=D\left(\tilde{m}_{3}, \tilde{f}_{3}(x)\right)=x \\
D\left(\tilde{M}_{1}, \tilde{f}_{1}(x)\right)=D\left(\tilde{M}_{3}, \tilde{f}_{3}(x)\right)=1-x, D\left(\tilde{m}_{2}, \tilde{f}_{2}(x)\right)=x^{2}, D\left(\tilde{M}_{2}, \tilde{f}_{2}(x)\right)=1-x^{2}
\end{gathered}
$$

so,

$$
\begin{gathered}
H_{1}(x)=H_{3}(x)=\frac{x}{(1+x)(2-x)}, \\
H_{2}(x)=\frac{x^{2}}{\left(1+x^{2}\right)\left(2-x^{2}\right)},
\end{gathered}
$$

It is easy to see that we have three supporting objectives (even though the functions of the modal values of the first two objectives are in conflict with the function of modal values of the third objective function). Therefore, from (10) we have $\Delta\left(\tilde{f}_{i}\right)=2, i=1,2,3$ and (11) turns into the following single objective problem

$$
\begin{gathered}
\min \left\{\left(\frac{x}{(1+x)(2-x)}\right)^{1 / 3},\left(\frac{x^{2}}{\left(1+x^{2}\right)\left(2-x^{2}\right)}\right)^{1 / 3},\left(\frac{x}{(1+x)(2-x)}\right)^{1 / 3}\right\} \rightarrow \max \\
\text { subject to } 0 \leq x \leq 1 .
\end{gathered}
$$

which has unique solution $x^{*}=1$, i.e. we have managed to reach the desired levels.

\section{Conclusions}

There has been a development over time in multiple criteria optimisation methods (cf. [19]). The first stage, the multiple attribute utility theory and the utility theory based multiple objective linear programming methods, aimed at aggregating preferences to a unique function, which then should be optimised. Thus the main focus was on exploring the mathematical conditions for a consistent aggregation, the forms of the aggregating functions and the methods for constructing mathematically useful preference structures with some fairly strong assumptions on the rationality of the decision maker. The second stage, which is overlapping with the first stage, was developed around the outranking relation [14], which represents the decision maker's preferences in a studied context when he has a certain set of information available. This outranking relation is assumed neither complete nor transitive and is thus much more realistic than the multiple attribute utility theory; it has also turned out to be quite useful for actual problem solving [19]. The third stage has developed around interactive methods, which combine successive optimization steps to find optimal compromise solutions with a dialogue with the decision maker to extract more information on his preferences. At its best an interactive method will serve as a searching 
and/or learning instrument in the problem space, which will help the decision maker to both formulate his preferences and to find an optimal solution.

Fuzzy set theory has proved to offer several distinct advantages as a basis for multiple criteria optimisation. It has been the basis for new and rather unconventional solution concepts, which are based on searching for some optimal option, which "best satisfies most of the important objectives", and differ significantly from the traditional notion to try to find an optimal option which best satisfies "all the objectives". It has turned out that the problem of conflicting objectives can be resolved, as a fuzzy conflict in a multiple objective programming model is not absolute and thus can be used as a basis for compromise solutions. There are also some practical advantages: when the solution derived from a well-formulated mathematical MCDM-model is applied to an actual problem there are some major problems to consider: (i) the set of feasible decision alternatives is fuzzy, and this set changes during the problem solving process; (ii) the decision maker does not always exist as an active entity, and the preferences consist of badly formulated beliefs, which are riddled with conflicts and contradictions; (iii) data on preferences are imprecise, and (iv) a decision should be good or bad not only in relation to some model, but in relation to the actual context. Here we have enhanced the multiple criteria optimization instruments with a technique for dealing with interdependence among the criteria. We have introduced a family of application functions which will help a decision maker to express his preferences, when any chosen preference is either conflicting or supportive in relation to any other preference.

\section{References}

[1] Carlsson, C. and Korhonen, P., A Parametric Approach to Fuzzy Linear Programming, Fuzzy Sets and Systems, 20(1986), 17-33

[2] C.Carlsson, Approximate Reasoning for solving fuzzy MCDM problems, Cybernetics and Systems: An International Journal, 18(1987), 35-48.

[3] C.Carlsson, Approximate Reasoning through Fuzzy MCDM-Methods, Operational Research'87, North-Holland, 1987 817-828.

[4] C.Carlsson, On interdependent fuzzy multiple criteria, in: R.Trappl ed.,Proceedings of the Eleventh European Meeting on Cybernetics and Systems Research, World Scientific Publisher, London, 1992, Vol.1. 139-146.

[5] C.Carlsson, On optimization with interdependent multiple criteria, in: R.Lowen and M.Roubens eds., Fuzzy Logic: State of the Art, Kluwer Academic Publishers, Dordrecht, 1992.

[6] C.Carlsson and R.Fullér, Multiple Criteria Decision Making: The Case for Interdependence, Compuetrs \& Operations Research, 22(1995) 251-260.

[7] M.Delgado,J.L.Verdegay and M.A.Vila, A possibilistic approach for multuobjective programming problems. Efficiency of solutions, in: R.Słowinski and J.Teghem eds., Stochastic versus Fuzzy Approaches to Multiobjective Mathematical Programming under Uncertainty, Kluwer Academic Publisher, Dordrecht, 1990 229-248.

[8] R.Felix, Multiple attribute decision making based on fuzzy relationships between objectives, in: Proceedings of the 2nd International Conference on Fuzzy Logic and Neural Networks, Iizuka Japan, July 17-22, 1992 805-808.

[9] R.Goetschel and W.Voxman, Elementary fuzzy calculus, Fuzzy Sets and Systems, 18(1986) 31-43. 
[10] O.Kaleva, Fuzzy differential equations, Fuzzy Sets and Systems, 24(1987) 301-317.

[11] J.Kacprzyk and R.R.Yager, Using fuzzy logic with linguistic quantifiers in multiobjective decision making and optimization: A step towards more human-consistent models, in: R.Slowinski and J.Teghem eds., Stochastic versus Fuzzy Approaches to Multiobjective Mathematical Programming under Uncertainty, Kluwer Academic Publishers, Dordrecht, 1990 331-350.

[12] E.Stanley Lee and R.J.Li, Fuzzy multiple objective programming and compromise programming with Pareto optimum, Fuzzy Sets and Systems, 53(1993) 275-288.

[13] H.Rommelfanger, FULP - A PC-supported procedure for solving multicriteria linear programming problems with fuzzy data, in: M. Fedrizzi, J.Kacprzyk and M.Roubens eds., Interactive Fuzzy Optimization, Springer-Verlag, Berlin, 1991 154-167.

[14] B.Roy, Decision-Aid and Decision-Making, in Bana e Costa ed., Readings in Multiple Criteria Decision Aid, Springer Verlag, 1990 17-35.

[15] M.Sakawa and H.Yano, Interactive Decision Making for Multiobjective Non-linear Programming Problems, Fuzzy Sets and Systems, 1989 315-326

[16] M.Sakawa and H.Yano, Feasibility and Pareto Optimality for Multiobjective Linear Programming Problems with Fuzzy Decision Variables and Fuzzy Parameters, in: J.Trappl ed., Cybernetics and Systems'90, World Scientific Publisher, London, 1990 155-162

[17] M.Sakawa and H.Yano, Interactive Decision Making for Multiobjective Linear Fractional Programming Problems with Fuzzy Parameters Based on Solution Concepts Incorporating Fuzzy Goals, Japanese Journal of Fuzzy Theory and Systems, 3(1991) 45-62.

[18] B.Schweizer and A.Sklar, Associative functions and abstract semigroups, Publ. Math. Debrecen, 10(1963) 69-81.

[19] P.Vincke, Multicriteria Decision Aid, John Wiley \& Sons, Chichester, 1992.

[20] E.Takeda and T.Nishida, Multiple Criteria Decision Problems with Fuzzy Domination Structures, Fuzzy Sets and Systems, 3(1980) 123-136.

[21] R.R.Yager, On ordered weighted averaging aggregation operators in multicriteria decisionmaking, IEEE Transactions on Systems, Man, and Cybernetics, 18(1988) 183-190.

[22] R.R.Yager, Family of OWA operators, Fuzzy Sets and Systems, 59(1993) 125-148.

[23] M.Zeleny, Multiple Criteria Decision Making, McGraw-Hill, New-York, 1982.

[24] H.-J.Zimmermann, Fuzzy programming and linear programming with several objective functions, Fuzzy Sets and Systems, 1(1978) 45-55.

[25] H.-J.Zimmermann, Decision Making in Ill-Structured Environments and with Multiple Criteria, in Bana e Costa ed., Readings in Multiple Criteria Decision Aid, Springer Verlag, 1990 119-151. 


\section{Follow ups}

The results of this paper have been improved and/or generalized in the following works.

\section{in journals}

A20-c30 Chi-Cheng Huang and Pin-Yu Chu, Using the fuzzy analytic network process for selecting technology R\&D projects, INTERNATIONAL JOURNAL OF TECHNOLOGY MANAGEMENT, 53(2011), number 1, pp. 89-115. 2011

http://inderscience.metapress.com/link.asp?id=u58122rt2768qx54

A20-c29 Jih-Jeng Huang, Chin-Yi Chen, Hsiang-Hsi Liu, Gwo-Hshiung Tzeng, A multiobjective programming model for partner selection-perspectives of objective synergies and resource allocations, EXPERT SYSTEMS WITH APPLICATIONS, 37(2010), Issue 5, pp. 3530-3536. 2010

http://dx.doi.org/j.eswa.2009.09.044

Carlsson and Fullér $(1994,1996)$ first proposed two methods to reshape the membership function for considering the problem of multiobjective programming with interdependence. Thereafter, several issues have been proposed to consider further situations such as uncertainty environment (Carlsson \& Fullér, 1996) and temporal interdependence (Östermark, 1997). However, several short-comings of their methods should be modified for considering the problem of partner selection in this paper. The first method (Carlsson \& Fullér, 1995), proposed by Carlsson and Fullér, does not precisely measure the supportive or the conflicting degree between the objectives and can only deal with the one-dimensional decision space. In contrast, the second method (Carlsson \& Fullér, 2002) can only be employed in the linear case. It can be seen that, the precise objective synergies is important for firms to choose partners and the objectives in firms or alliances are usually complex and nonlinear functions. Therefore, a more accurate and flexible index should be given in order to choose the best partners in alliances. (page 3532)

A20-c28 XH Yu, ZS Xu, Hierarchical Aggregation Methods Based on Weighted Combination Operators, INFORMATION-AN INTERNATIONAL INTERDISCIPLINARY JOURNAL, 12(2009), Issue 1, pp. 51-64. 2009

A20-c27 Fuzhan Nasiri, Gordon Huang, A fuzzy decision aid model for environmental performance assessment in waste recycling, Environmental Modelling \& Software 23 (2008) 677-689. 2008 http://dx.doi.org/10.1016/j.envsoft.2007.04.009

A20-c26 Zhang Ling, Multi-attribute decision making based on association theory research, MANAGEMENT REVIEW, 20(2008), number 5, pp. 51-57 (in Chinese). 2008

http://www.cqvip.com/qk/96815a/2008005/27274741.html

A20-c25 Chang JR, Cheng CH, Chen LS, A fuzzy-based military officer performance appraisal system, APPLIED SOFT COMPUTING 7 (3): 936-945 JUN 2007

http://dx.doi.org/10.1016/j.asoc.2006.03.003 
Carlsson and Fullér [A19, A20] introduced the concept of interdependence in multiple criteria decision making (MCDM), and some researchers showed that fuzzy set theory [28] could be successfully applied to resolve multiple criteria problems [10,23,26,27]. In general, the appraisal from among two or more people is a multiple criteria decision making problem. Under many situations, the values for the qualitative criteria are often imprecisely defined for the decision makers. (page 937)

A20-c24 Nasiri F, Maqsood I, Huang G, et al., Water quality index: A fuzzy river-pollution decision support expert system, JOURNAL OF WATER RESOURCES PLANNING AND MANAGEMENT - ASCE, 133 (2): 95-105 MAR-APR 2007

http://dx.doi.org/10.1061/(ASCE)0733-9496(2007)133:2(95)

A20-c23 Gal T, Hanne T Nonessential objectives within network approaches for MCDM EUROPEAN JOURNAL OF OPERATIONAL RESEARCH, 168 (2): 584-592 JAN 162006

http://dx.doi.org/10.1016/j.ejor.2004.04.045

A20-22 Myung HC, Bien ZZ, Design of the fuzzy multiobjective controller based on the eligibility method, INTERNATIONAL JOURNAL OF INTELLIGENT SYSTEMS, 18 (5): 509-528 MAY 2003

http://dx.doi.org/10.1002/int.10101

Define the relation between two objectives as follows [A20]:

1. $f_{i}$ supports $f_{j}$ if $f_{i}\left(u\left(t_{1}\right), x\left(t_{1}\right)\right) \geq f_{i}\left(u\left(t_{2}\right), x\left(t_{2}\right)\right)$ entails $f_{j}\left(u\left(t_{1}\right), x\left(t_{1}\right)\right) \geq$ $f_{j}\left(u\left(t_{2}\right), x\left(t_{2}\right)\right)$, for $u \in U$ and $t_{1} \leq t_{2}$,

2. $f_{i}$ conflicts $f_{j}$ if $f_{i}\left(u\left(t_{1}\right), x\left(t_{1}\right)\right) \geq f_{i}\left(u\left(t_{2}\right), x\left(t_{2}\right)\right)$ entails $f_{j}\left(u\left(t_{1}\right), x\left(t_{1}\right)\right)<$ $f_{j}\left(u\left(t_{2}\right), x\left(t_{2}\right)\right)$, for $u \in U$ and $t_{1} \leq t_{2}$,

3. $f_{i}$ and $f_{j}$ are independent, otherwise

(page 518)

A20-c21 Lei, X., Shi, Z. Overview of multi-objective optimization methods, JOURNAL OF SYSTEMS ENGINEERING AND ELECTRONICS, 15 (2), pp. 142-146. 2004

A20-c20 Matthias Ehrgott and Stefan Nickel, On the number of Criteria Needed to Decide Pareto Optimality, MATHEMATICAL METHODS OF OPERATIONS RESEARCH, 55(2002) 329-345. 2002

http://dx.doi.org/10.1007/s001860200207

A more general concept of interdependent criteria has been discussed in [A19], see also [A20]. Our approach is related to this topic in the sense that we determine the number of objectives which are necessary to prove Pareto optimality for a given point. However, the theory presented in this paper is more general: the results also hold in the absence of nonessential criteria, as will be ... (page 330)

A20-c19 Gal T, Hanne T, Consequences of dropping nonessential objectives for the application of MCDM methods, EUROPEAN JOURNAL OF OPERATIONAL RESEARCH, 119(2): 373-378 DEC 11999

http://dx.doi.org/10.1016/S0377-2217(99)00139-3 
The problem of obtaining well-designed criteria for a multiple criteria decision making problem is well known (see e.g. Bouyssou, 1992; Keeney and Raiffa, 1976, pp. 5053; Keeney, 1992, pp. 82-87, 120; Roy, 1977; Roy and Vincke, 1984). However, the problem of interdependence among the criteria is seldom treated in the literature (Carlsson and Fullér, 1995; see also Carlsson and Fullér, 1994). (page 373)

A20-c18 Jonathan Lee, Jong-Yih Kuo, New approach to requirements trade-off analysis for complex systems IEEE TRANSACTIONS ON KNOWLEDGE AND DATA ENGINEERING, 10 (4), pp. 551-562 JUL-AUG 1998

http://dx.doi.org/10.1109/69.706056

Carlsson and Fullér [A20] propose an approach to fuzzy multiple objective programming (FMOP) with interdependency relationships among objectives, which is an extension of Carlsson's MOP [4] to fuzzy logic. Three kinds of relationships have been identified: supportive, conflicting, and independent. The basic idea is to utilize these relationships to modify the membership function of the so called good solution. Felix [15] and Felix et al. [17] propose an approach, called DMRG (Decision Making Based on Relationship between Goals), to defining a spectrum of relationships based on fuzzy inclusion and fuzzy noninclusion: independent, assist, cooperate, analogous, hinder, compete, trade-off, and unspecified dependent, and to determining the final set of decision alternatives according to the relationships. These approaches are similar to ours in two aspects: the problems of modeling the relationships, and the issues of aggregation.(page 558)

A20-c17 Jonathan Lee, Jong-Yih Kuo, Fuzzy decision making through trade-off analysis between criteria, INFORMATION SCIENCES, 107(1998) 107-126. 1998

http://dx.doi.org/10.1016/S0020-0255(97)10020-2

In addition, Carlsson and Fullér [A20] advocated that much closer to MCDM in the real world than the traditional MCDM are the cases with interdependent criteria. However, current relationship analysis approaches (e.g. fuzzy multiple objective programs (FMOP) [A20] and decision making based on relationship between goals (DMRG) $[2,4,5])$ usually result in identifying relationships that are contradictory to each other. (page 108)

Recently, Carlsson and Fullér [A20] proposed an approach to FMOP with interdependency relationships among objectives, which is an extension of Carlsson's MOP [20] to fuzzy logic. The basic idea is to utilize these relationships to modify the membership function of the so-called 'good solution', denoted as $H_{i}$. (page 121)

A20-c16 J. Tang and D. Wang, An interactive approach based on a genetic algorithm for a type of quadratic programming problems with fuzzy objectives and resources, COMPUTERS \& OPERATIONS RESEARCH, 24(1997) 413-422. 1997

http://dx.doi.org/10.1016/S0305-0548(96)00059-7

The current research on fuzzy mathematical programming was largely limited in the range of linear programming [10-12] and multiobjective programming [13, A20, 15], but fuzzy nonlinear programming [16] including fuzzy quadratic programming is rarely involved. (page 414) 
A20-c15 R. Östermark, Temporal interdependence in fuzzy MCDM problems, FUZZY SETS AND SYSTEMS, 88(1997) 69-79. 1997

http://dx.doi.org/10.1016/S0165-0114(96)00046-2

The case with unknown parameter values, e.g., future sales prices, interest rates etc. is not considered in the present study. Compared to the static concept of Carlsson and Fullér [A20], we have set of $k$ objective function trajectories defined over the planning horizon, not merely $k$ (static) objective function values. (page 71)

In this study we have considered temporal interdependence in multiple criteria decision making. Our analysis extends the (static) concept introduced by Carlsson and Fullér [A20] in a way that allows coping with goal conflicts typically arising in managerial decisioin making. The concept of temporal interdependence was used to describe mutually supportive and mutually conflicting criteria in a multiperiod firm model. Next, the static membership function proposed by Carlsson and Fullér [A20] was generalized to the dynamic case both for DMOP and DFMOP problems. We showed that incorporating the discount rate of the firm, i.e., the risk-adjusted weighted average cost of capital in the dynamic objective functions is essential in corporate planning. The cost of capital affects the shape of the membership functions and, therefore, the mutual support vs. conflict in the objective set. In the derivations we have utilized the simplifying assumption that all objectives are equally important. This allows usage of the number of criteria as a measure of support/conflict in the objective set, precisely as in Carlsson and Fullér [A20]. (page 78)

\section{in proceedings and in edited volumes}

A20-c13 Weiyi Qian, An inexact approach based on Genetic Algorithm for fuzzy programming problems, Sixth International Conference on Natural Computation (ICNC), 10-12 August 2010, Yantai, China, [ISBN 978-1-4244-5958-2], pp. 2281-2285. 2010

http://dx.doi.org/10.1109/ICNC.2010.5584212

A20-c12 Z.K. Öztürk, A review of multi criteria decision making with dependency between criteria, 18th International Conference on Multiple Criteria Decision Making, June 19-23, 2006, Chania, Greece. 2006

http://citeseerx.ist.psu.edu/viewdoc/summary?doi=10.1.1.98.1782

A20-c11 Zhang, L., Zhou, D., Zhu, P., Li, H. Comparison analysis of MAUT expressed in terms of choquet integral and utility axioms 1st International Symposium on Systems and Control in Aerospace and Astronautics, 2006, art. no. 1627708, Jan. 19-21, 2006, Harbin, China, pp. 85-89. 2006

http://dx.doi.org/10.1109/ISSCAA.2006.1627708

Carlsson and Fullér demonstrated that the use of interdependences among objectives of MCDM provides for more correct solutions and faster convergence [A18, A19, A20]. (page 85) 
A20-c10 Jong-Yih Kuo and Jonathan Lee, Evolution of Intelligent Agent in Auction Market, in: FUZZY IEEE 2004 CD-ROM Conference Proceedings Budapest, July 26-29, 2004, IEEE Catalog Number: 04CH37542C, [ISBN 0-7803-8354-0], pp. 331-336 (file name: 0052-1350.pdf). 2004

http://ieeexplore.ieee.org/iel5/9458/30040/01375744.pdf?

Most of the existing approaches in multiple criteria making lack the aspect of an explicit modeling of relationships between goals. As was pointed out by Felix [8], a few of the existing MCDM approaches refer to the aspect of an explicit modeling of relationships between goals. Carlsson and Fullér [A20] advocated that much closer to MCDM in the real world than the traditional MCDM are the case with interdependent criteria. However, current relationship analysis approaches (e.g. [A20], [9]) usually result in identifying relationships that are contradictory to each other. Our previous work on Criteria Trade-off Analysis has been on the formulation of soft criteria based on Zadeh's canonical form in test-score semantics and an extension of the notion of soft condition [14]. The trade-off among soft goals is analyzed by identifying the relationships between goals. A compromise overall satisfaction degree can be obtained through the aggregation of individual goal based on the goals hierarchy. (page 334)

A20-c9 Xin-Wang Liu Qing-Li Da Liang-Hua Chen, A note on the interdependence of the objectives and their entropy regularization solution, Proceedings of the Second International Conference on Machine Learning and Cybernetics, Xi'an, 2-5 November 2003, [ISBN: 0-7803-7865-2], pp. 2677- 2682. 2003

A20-c8 Jonathan Lee, Jong-Yih Kuo et al., Trade-off Requirement Engineering, in: Jonathan Lee ed., Software Engineering with Computational Intelligence Series: Studies in Fuzziness and Soft Computing, Vol. 121 Springer, [ISBN: 978-3-540-00472-1] 2003 pp. 51 -71. 2003

A20-c7 Myung, H.-C., Bien, Z.Z. Interdependent multiobjective control using Biased Neural Network (Biased NN) Annual Conference of the North American Fuzzy Information Processing Society NAFIPS, 3, pp. 1378-1383. 2001

http://ieeexplore.ieee.org/iel5/7506/20427/00943750.pdf?arnumber=943750

A20-c6 Matthias Ehrgott and Stefan Nickel, On the number of Criteria Needed to Decide Pareto Optimality, WIMA Report, Fachbereich Mathematik, Universität Kaiserslautern, No. 2, 1999.

A more general concept of interdependent criteria has been discussed in [A19], see also [A20]. Our approach is related to this topic in the sense that we determine the number of objectives which are necessary to prove Pareto optimality for a given point. However, the theory presented in this paper is more general: the results also hold in the absence of nonessential criteria, as will be ...

A20-c5 Didier Dubois and Henri Prade, Fuzzy criteria and fuzzy rules in subjective evaluations - a general discussion. In Proc. 5th European Congress on Intelligent Technologies and soft Computing (EUFIT 97), September 8-12, 1997, Aachen, Germany, 975-978. 1997

ftp://ftp.irit.fr/pub/IRIT/RPDMP/FCFRSE.ps.gz

Such an approach raises several questions about 
- the choice of proper scales (what kind? qualitative or numerical scale?), their commensurability, and the meaningfulness of the aggregation operations w.r.t. the scale;

- the practical elicitation of the membership functions, and of the appropriate operations (compensatory, or purely logical conjunctions, for instance); see (Dubois and Prade, 1988) on this latter point, where the elicitation of aggregation operations is based on the knowledge of the decision's maker's behavior in well-contrasted situations;

- the modelling of the importance (by means of weights or thresholds) of the criteria, and more generally of the interaction between criteria (Carlsson and Fullér, 1994; Grabisch, 1997).

(page 976)

A20-c4 Lee J, Jong-Yih Kuo, Huang W T Fuzzy decision making through relationships analysis between criteria In: Fuzzy Systems Symposium, 1996. 'Soft Computing in Intelligent Systems and Information Processing', 11-14 December 1996, Kenting, Taiwan, pp. 296-301. 1996

http://dx.doi.org/10.1109/AFSS.1996.583617

Current relationships analysis approaches such as FMOP (fuzzy multiple objective programs) [A20] and DMRG (decision making based on relationships between goals), however, usually result in identifying relationships between criteria that are contradictory to each other. Furthermore, the aggregation operators selected in their aggregation procedures either derive more than one alternative or fail to come up with any. (page 296)

\section{in books}

A20-c2 S.N. Sivanandam and S. N. Deepa. Introduction to Genetic Algorithms, Springer, [ISBN 9783540731894], 2007.

A20-c1 G. J. Klir and B. Yuan, Fuzzy Sets and Fuzzy Logic: Theory and Applications, Prentice Hall, [ISBN 0-13-101171-5], 1995.

\section{in Ph.D. dissertations}

- Sudaryanto, A fuzzy multi-attribute decision making approach for the identification of the key sectors of an economy: The case of Indonesia, RWTH Aachen Germany. 2003

http://darwin.bth.rwth-aachen.de/opus3/volltexte/2003/591/ 